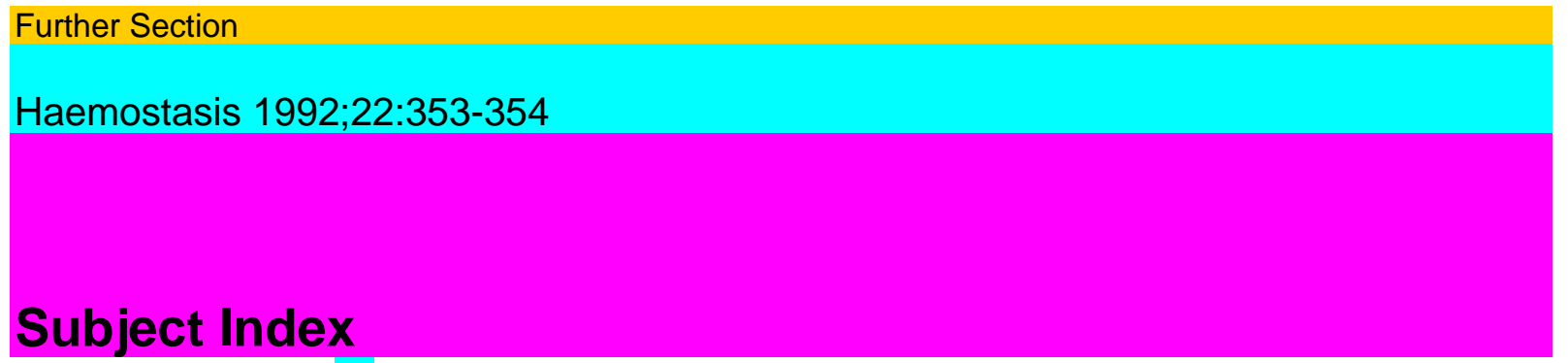

Activated protein C 165 Acute ischemic stroke 99

- $\quad$ leukemia 117, 216

Aggregation 138

AIDS 281,299

Alcoholic liver disease 340

Animal model 322

Anticoagulation 129

Anti-idiotype 268

Antithrombin III 12, 17,66, 153,

340 Antithrombotic agents 322

compound 202

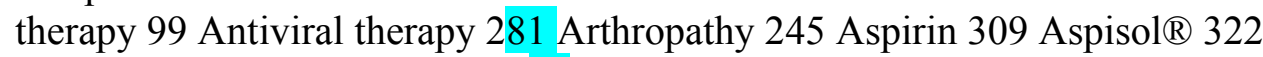

Bleeding models 58 Blood clot 195

Cerebral hemorrhage 259

Cicaprost 322

Clexane 85

Clot retraction 195

Coagulation defects 309

Comprehensive care 247, 299,

305 Counterion 113 Cross-reactivity 85 Crotalus molossus molossus

venom 41 Cryoprecipitate infusion 233

Danazol 12

Z)-Dimer 117

Decline, CD4 cells 25

Deep vein thrombosis 92, 104

laxis 211

venous thrombosis, prophy

Desmopressin 276 Diabetes 187

- mellitus 334

Diabetic nephropathy 334

Diagnosis 236

Dialyzer 12 Differentiation 173 Disseminated intravascular coagulation 17, 179 Dosing regimen

73 Dysfibrinogenemia 7

Early management 99

Effects on hemostatic system in

vitro 153 Elastase 216 Elective hip surgery 109 Endothelial injury 202 Epinephrine 138

Erythropoietin 12 Expert panel 221

Factor IV immunoglobulin 268

Va 165 
VIII 245, 268

- concentrates 25

IXa 66

Xa 58,66 Factors VII and X 340 FEIBA 268

Fibrin degradation 41 Fibrinogen degradation 41 variant 7

Fibrinolysis 12, 41, 117, 187, 195 Fibrinopeptide A 7,153 Financial support 293 Flow cytometry 32 Fragmin 85 Fraxiparine ${ }^{\circledR} 85,322$

Glanzmann's thrombasthenia 330 Glycoprotein lib 330

ПIb-IIIa 32

Ilia 330

H(a)emophilia 25,259,276,281, 293,299,305

- A 251

-, home therapy 247

-, Spanish Federation 305

- $\quad$ centers 299

H(a)emophiliacs 245 Haemorrhage 92 Haemorrhagic diathesis 221 Head injuries 259

Hemodialysis 12 Heparin 58,66, 129, 153,309 Heparin-induced thrombocyto-

penia 85 Heparinoid, low molecular weight

73 Hepatitis, HIV infection 305 Heredity 236 Hip fracture 104 Hirudin 309 History taking 221

HIV infection 259, $293 \mathrm{HIV}-1$ infection $15 \mathrm{HN}-11501322$

Immunoblotting 330 Immunoelectrophoresis 32 Immunological tolerance 268

Immunosuppression 268 Impedance-based cell counter 160 Interferon- $\gamma 173$ Intraduodenal absorption 113 Isch(a)emic heart disease 138

- $\quad$ stroke 92

\section{KRDS 1}

Laser 147,322 Long-term therapy 251 Low-molecular-weight heperin 113

salt 113

heparinoid 92, 109

Macrophages 173

Magnetic resonance imaging 195

Microthrombus formation 322

Molsidomine 322

Monoclonal antibodies 330

Monocytes 173

Myocardial infarction 187

353

Native whole blood 153 Necrosis 124

Observer variation 211 On-demand' prophylaxis 245

- $\quad$ treatment 251

Orgaran(Org 10172) 58,66,73,

$85,92,99,104$ Orthopaedic score 251

Patient interview 221 PCA-4230 202 Pentasaccharide 309 Pharmacokinetics 73 Plasmin activity

179 Plasmin-c $1 / 8$-antiplasmin complex

179 Plasminogen activator inhibitor

12, 187 Platelet(s) 1, 138,147, 160,330 
activation 202

factor 4153

function 334

transfusion 233

von Willebrand factor 233 Platelet-induced thrombin generation 309

Porcine factor VIII 268 Pregnancy 160

Prognostic markers 281 Prophylaxis 92, 104 Prostaglandin E[ 153 Protein C 12,340

S 12, 165 Proteolysis 216 Prothrombin fragment 1+2 17

time 340 Prothrombinase 66 Psychologic stress 138

Rat 147

Recombinant activated factor VII

268 Russell's viper venom 153

Scandinavia 236

Self-treatment 247

Sepsis 17

Socio-economic benefit 247

Spinal hemorrhage 259

Spontaneous platelet aggregation

160 Substitution therapy 305 Surgery 211

Thrombin 58, 66

- $\quad$ activity 179

Thrombin-antithrombin-III

complex 179 ß-Thromboglobulin 334 Thrombophlebitis 129 Thromboplastin 173 Thrombosis 1 , $12,124,147$

models 58

prophylaxis 73, 109 Thromboxane 138

B2 334 Ticlopidine 147,202 Tissue factor 173

plasminogen activator 187 Tumor cells 165

Type III von Willebrand disease 233

Vascular access 12 Venography 211 Venous thrombosis 129 von Willebrand disease 276

- -, type I and III 236

- factor 32

Warfarin 124

World Federation of Hemophilia

293

Yolk immunoglobulins 32

354

Subject Index 